\title{
Assessment of a Discontinuous Galerkin Method for the Simulation of the Turbulent Flow around the DrivAer Car Model
}

\author{
Alessandro Colombo ${ }^{1}\left(\mathbb{D}\right.$, Andrea Bortoli ${ }^{2}$, Pierangelo Conti ${ }^{2}$, Andrea Crivellini ${ }^{3}{ }^{(D}$, Antonio Ghidoni ${ }^{4} \oplus^{\circ}$, \\ Alessandra Nigro ${ }^{3}$ (D) and Gianmaria Noventa ${ }^{4, *}$ (D) \\ 1 Department of Engineering and Applied Sciences, University of Bergamo, Viale Marconi 5, \\ 24044 Dalmine, Italy; alessandro.colombo@unibg.it \\ 2 University of Bergamo, Viale Marconi 5, 24044 Dalmine, Italy; pierangelo.conti@unibg.it \\ 3 Department of Industrial Engineering and Mathematical Science, Marche Polytechnic University, via Brecce \\ Bianche 12, 60131 Ancona, Italy; a.crivellini@staff.univpm.it (A.C.); a.nigro@staff.univpm.it (A.N.) \\ 4 Department of Mechanical and Industrial Engineering, University of Brescia, via Branze 36, \\ 25123 Brescia, Italy; antonio.ghidoni@unibs.it \\ * Correspondence: gianmaria.noventa@unibs.it
}

Citation: Colombo, A.; Bortoli, A.; Conti, P.; Crivellini, A.; Ghidoni, A.; Nigro, A.; Noventa, G. Assessment of a Discontinuous Galerkin Method for the Simulation of the Turbulent flow around the DrivAer Car Model. Appl. Sci. 2021, 11, 10202. https:// doi.org/10.3390/app112110202

Academic Editor: Artur Tyliszczak

Received: 11 October 2021

Accepted: 28 October 2021

Published: 31 October 2021

Publisher's Note: MDPI stays neutral with regard to jurisdictional claims in published maps and institutional affiliations.

Copyright: (c) 2021 by the authors. Licensee MDPI, Basel, Switzerland. This article is an open access article distributed under the terms and conditions of the Creative Commons Attribution (CC BY) license (https:/ / creativecommons.org/licenses/by/ $4.0 /)$.

\begin{abstract}
The turbulent flow over the DrivAer fastback model is here investigated with an orderadaptive discontinuous Galerkin (DG) method. The growing need of high-fidelity flow simulations for the accurate determination of problems, e.g., vehicle aerodynamics, promoted research on models and methods to improve the computational efficiency and to bring the practice of Scale Resolving Simulations (SRS), like the large-eddy simulation (LES), to an industrial level. An appealing choice for SRS is the Implicit LES (ILES) via a high-order DG method, where the favourable numerical dissipation of the space discretization scheme plays directly the role of a subgrid-scale model. Implicit time integration and the $p$-adaptive algorithm reduce the computational cost allowing a high-fidelity description of the physical phenomenon with very coarse mesh and moderate number of degrees of freedom. Two different models have been considered: (i) a simplified DrivAer fastback model, without the rear-view mirrors and the wheels, and a smooth underbody; (ii) the DrivAer fastback model, without rear-view mirrors and a smooth underbody. The predicted results have been compared with experimental data and CFD reference results, showing a good agreement.
\end{abstract}

Keywords: car aerodynamics; DrivAer model; discontinuous Galerkin; Implicit LES; orderadaptive method

\section{Introduction}

In the last decade, the Computational Fluid Dynamics (CFD) has received increasing attention from the research community and automotive industry, as it is considered an important design tool that can supplement the more expensive experiments in wind tunnels. The accurate prediction of the complex massively separated turbulent flow around a car is becoming of paramount importance to reduce noise level and improve fuel efficiency. As observed in [1], the aerodynamic drag is the main energy-consumption source over $80 \mathrm{~km} / \mathrm{h}$. Moreover, the effect of the aerodynamic drag on energy losses is amplified in electric vehicles, where losses are 4.4 times larger than in vehicles with internal combustion engines [2]. The prediction of the lift and drag forces has been conventionally carried out with the Reynolds Averaged Navier Stokes (RANS) approach, which allows one to obtain reference values at a feasible computing cost for industry. However, the typical unsteady features of the flow around bluff and blunt ended bodies such as hatchback, estate and SUV type cars are difficult to predict with a RANS approach. Examples of reliable drag prediction by RANS can be found in literature, but they seem case dependent [3-6] and suggest avoiding RANS for an accurate aerodynamic development. To overcome 
these limitations, more accurate and costly turbulence approaches can be used, such as hybrid RANS-LES [6] or LES [7] methods, whose widespread diffusion in industry has been fostered by the ever increasing computational resources.

Realistic car models were not available for CFD simulations before 2012, and only simplified geometries such as the Ahmed [8] or the SAE [9] bodies could be used for validation and sensitivity studies. However, these models were often characterised by few aerodynamic features representative of a car. To fill the gap between a very simple bluff body and a fully detailed production car, TU Munich in collaboration with the Audi AG and the BMW Group designed and made public the so-called DrivAer car model [10]. The model is available with three different rear end configurations: fastback, estate back, and notchback. Each model is available with a smoothed and a detailed underbody, with/without the side mirrors and wheels.

Heft et al. [11] carried out for the first time simulations with $k-\omega$ SST and $k-\omega$ SAS SST turbulence models on the fastback geometry. These results predicted reasonably well the averaged flow field around the vehicle, while some limitations appear for the detached regions. These results strengthen the belief that Scale-Resolving Simulations (SRS) are needed to correctly describe unsteady flow phenomena around vehicles. Guilmineau et al. [4] in 2014 carried out RANS simulations with an anisotropic two-equation explicit algebraic Reynolds stress and DES models on the fastback geometry. DES guaranteed a higher accuracy than RANS simulations in the prediction of drag and lift coefficients and reproduced correctly the instantaneous vortical structures. Ashton et al. [6] compared different turbulence models for RANS simulation, e.g., $k-\epsilon, k-\omega$ SST, Spalart-Allmaras, B-EVM and EB-RSM, and DES-type models, on the fastback and estate geometry. RANS simulations were not able to correctly predict force coefficients and pressure distribution around the cars. In particular, DDES (delayed detached-eddy) and IDDES (improved delayed detached-eddy) were also considered to investigate in depth the prediction capability of the most representative hybrid methods. They showed clear advantages over RANS in the prediction of the force coefficients and unsteady flow features. However, some limitations are still evident to correctly capture the pressure distribution and vortical structures in the rear end of the car. In 2018 Aljure et al. [12] investigated the potential of the wall modelled large-eddy (WMLES) in simulating unsteady flow features over a realistic car model. WMLES proved to be able to predict unsteady flow features, but a detailed analyses on the vortical structures around the car is not provided. Recently, Rüttgers et al. [13] adopted a LES (Large Eddy Simulation) approach to predict the turbulent flow around the fastback geometry, providing a detailed and systematic analysis of the unsteady flow features.

In the present study an efficient order-adaptive discontinuous Galerkin (DG) method [14] for incompressible flows is used to perform the implicit LES [15] around the DrivAer fastback model. High-order DG finite elements methods proved to be very well suited [16-25] for ILES, where the unfiltered incompressible Navier-Stokes equations are solved, and the numerical dissipation introduced by the discretizaton itself, e.g., by numerical fluxes and the viscous stabilisation, plays the role of an explicit subgrid-scale (SGS) model that dissipates the smallest scale eddies.

The DG solver is characterised by (i) the local adaptation of the polynomial degree of the solution over the mesh, (ii) a multi-constraint domain decomposition algorithm to ensure the computational balance over the processes, (iii) the adaptation of the degree of exactness for quadrature rules and (iv) a $p$-MG multilevel preconditioner for the linear solver on the implicit time integration. Moreover, the non-trivial task of the high-order mesh generation is strongly simplified with the $p$-adaptive algorithm, as the local mesh refinements related to a priori knowledge of the flow features are avoided.

In particular, the aim of this work is to assess the feasibility of the ILES approach in the accurate simulation of the high-Reynolds turbulent flow around a simplified car model with a moderate number of degrees of freedom (DoFs). Two different models have been considered: (i) a simplified DrivAer fastback model, where the handles, the rear-view mirrors and the wheels have been removed, the underbody has been smoothed, and the 
front radiator grid has been closed; (ii) the DrivAer fastback model, where the handles and the rear-view mirrors were removed, and a smoothed underbody and a closed front radiator grids were considered to reduce the complexity of the "original" DrivAer model. The first model has been used to investigate the effect of the Reynolds number and of the absence of the wheels on the aerodynamic performance. Two different Reynolds number have been considered, $R e_{L}=2.435 \times 10^{6}$ and $R e_{L}=4.87 \times 10^{6}$, based on the model length $L$ and the freestream conditions $\left(u_{\infty}=8 \mathrm{~m} / \mathrm{s}\right.$ and $u_{\infty}=16 \mathrm{~m} / \mathrm{s}$, respectively). The second model has been used to assess the predicting capability of the proposed ILES approach with a moderate number of DoFs, comparing the results with experimental data and numerical results available in literature.

The paper is organised as follows. The present computational methodology is presented in Sections 2.1 and 2.2, while Section 2.3 gives some information about the mesh generation process. Sections 3.1 and 3.2 report the results for the two considered DrivAer geometries. Finally, the conclusions are given in Section 4.

\section{The Numerical Framework}

In this section the space and time discretizations of the incompressible Navier-Stokes equations and the main strategies used to speed-up the solution process are presented.

\subsection{Discontinuous Galerkin Method}

ILES simulations have been performed in this work, where the unfiltered NavierStokes equations are solved. As reported in literature [16-24], DG methods are well suited for ILES, due to their dissipation and dispersion properties. The numerical dissipation introduced by the discretizaton itself, e.g., by numerical fluxes and the viscous stabilization, mainly acts at the smallest under-resolved scales and resembles a high frequency filter that mimics the role of a sub-grid-scale model. The ILES of several canonical and complex flows have been performed with the present code and can be found in $[14,26]$.

An efficient order-adaptive incompressible discontinuous Galerkin (DG) method [14] has been used in this work. The incompressible DG solver relies on a modified formulation for the inviscid interface numerical flux [27], computed as the exact solution of the Riemann problem relaxed by an artificial compressibility perturbation that guarantees the necessary coupling between the discrete incompressibility constraint and the rest of the governing equations. The nonlinear system of differential algebraic equations arising from the spatial discretization is advanced in time using a high-order time integration scheme, i.e., the linearly-implicit Rosenbrock-type Runge-Kutta scheme, which overcomes the severe timestep restriction proper of explicit methods. In particular, the three-stage, order three ROS3P method [28] has been used. For a comprehensive discussion on the benefits of a high-order time integration scheme adopted in this work, see [29-31].

The main features of the DG solver are (i) the local adaptation of the polynomial degree of the solution over the mesh, (ii) a multi-constraint domain decomposition algorithm to ensure the computational balance over the processes, (iii) the adaptation of the degree of exactness for quadrature rules, and (iv) a $p$-MG multilevel preconditioner for the linear solver included in the implicit time integration.

\section{2. $p$-Adaptation Strategy}

Polynomial functions are adopted in DG methods to represent the numerical solution inside the elements of the mesh with no continuity requirements at the cells interfaces. As a consequence, the order of accuracy can be varied locally by using a different degree of the polynomial representation (see Figure 1) according to some error indicator. In literature this approach is known as $p$-adaptation [32-36] and allows for the capture of the flow features by changing locally the solution accuracy.

The time-averaged solution is used to compute the error estimator, as statistically stationary unsteady flow problems are the objective of this work. The estimator combines two contributions: (i) a measure of the solution jumps at grid cells interfaces [37,38]; (ii) the 
decay rate of the modal coefficients of the polynomial expansion [39]. Both indicators aim at revealing a lack/excess of spatial accuracy in the numerical discretization and are here applied to the pressure variable. For a comprehensive discussion on the error estimator implementation see [14]. This strategy can be used in parallel only with a balanced repartitioning of the computational grid after each adaptation cycle, to overcome the natural imbalance of floating point operations per domain induced by the adaptation. The flow chart of the solution strategy is reported in Figure 2. The adaptation process is triggered by a simple indicator, $\operatorname{trg}$, defined as the norm of the relative increment, computed at each time step, of the vector of the degrees of freedom of the time-averaged solution at run-time $\overline{\mathbf{U}}$. $l$ is the index denoting the $l$-th adaptive cycle, $i$ denotes the $i$-th time-step, while $j$ denotes the $j$-th iteration between two adaptation cycles. The user-defined parameters for the simulation are: the number of adaptation cycles $n_{a d p}$, the threshold tolerance for the activation of the adaptation process $t_{0} l_{\text {trg }}$ and the minimum number of time steps between two adaptation cycles $\mathcal{N}_{a d p}$. At each adaptation cycle, only $20 \%$ of elements with the higher estimated error are marked for refinement, while the coarsening is disabled. This choice is motivated by the use of coarse meshes for the simulations.
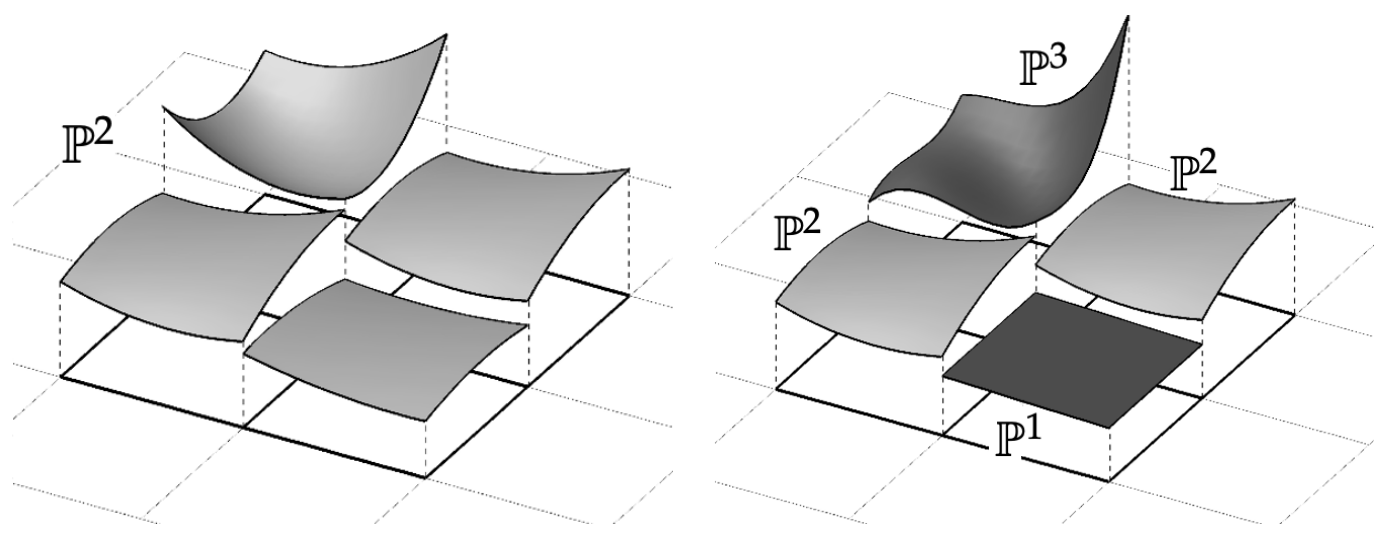

Figure 1. Comparison between a fixed (left) and variable (right) order approach to represent the solution.

The $p$ refinement allows also for the simplification of the mesh generation process, as the typical a priori mesh refinement required capturing the known flow features, such as wakes or detachments, is avoided. Moreover, in this work an approach to locally adapt the degree of exactness of quadrature rules according to the mesh characteristics is adopted, to avoid the over-integration of straight-sided elements, which appear in regions far from the walls. Finally, a multilevel preconditioner is used to strongly reduce the memory requirements and the operation count [26]. This preconditioner speeds up the linear systems solution, here performed via the memory-saving matrix-free FGMRES method, by solving coarse problems and moving along a given path, e.g., a V-cycle. These coarse problems are defined as a sequence of reduced polynomial degree discretizations and use GMRES as a smoother on each level. On each level, except the coarsest, the smoothers use a cheap matrix-based preconditioner built by a simply neglecting the off-diagonal blocks of the restricted implicit operator. All the details of this preconditioning technique and its use in the order adaptive framework are presented in [14,26].

\subsection{High-Order Mesh Generation}

An accurate treatment of curved boundaries is mandatory for the application of finite element methods such as DG methods to industrial CFD computations. In particular, highorder coarse meshes are needed to fully exploit the potential of high-order DG methods in delivering accurate solutions, while saving the overall number of cells needed to represent the geometry. 


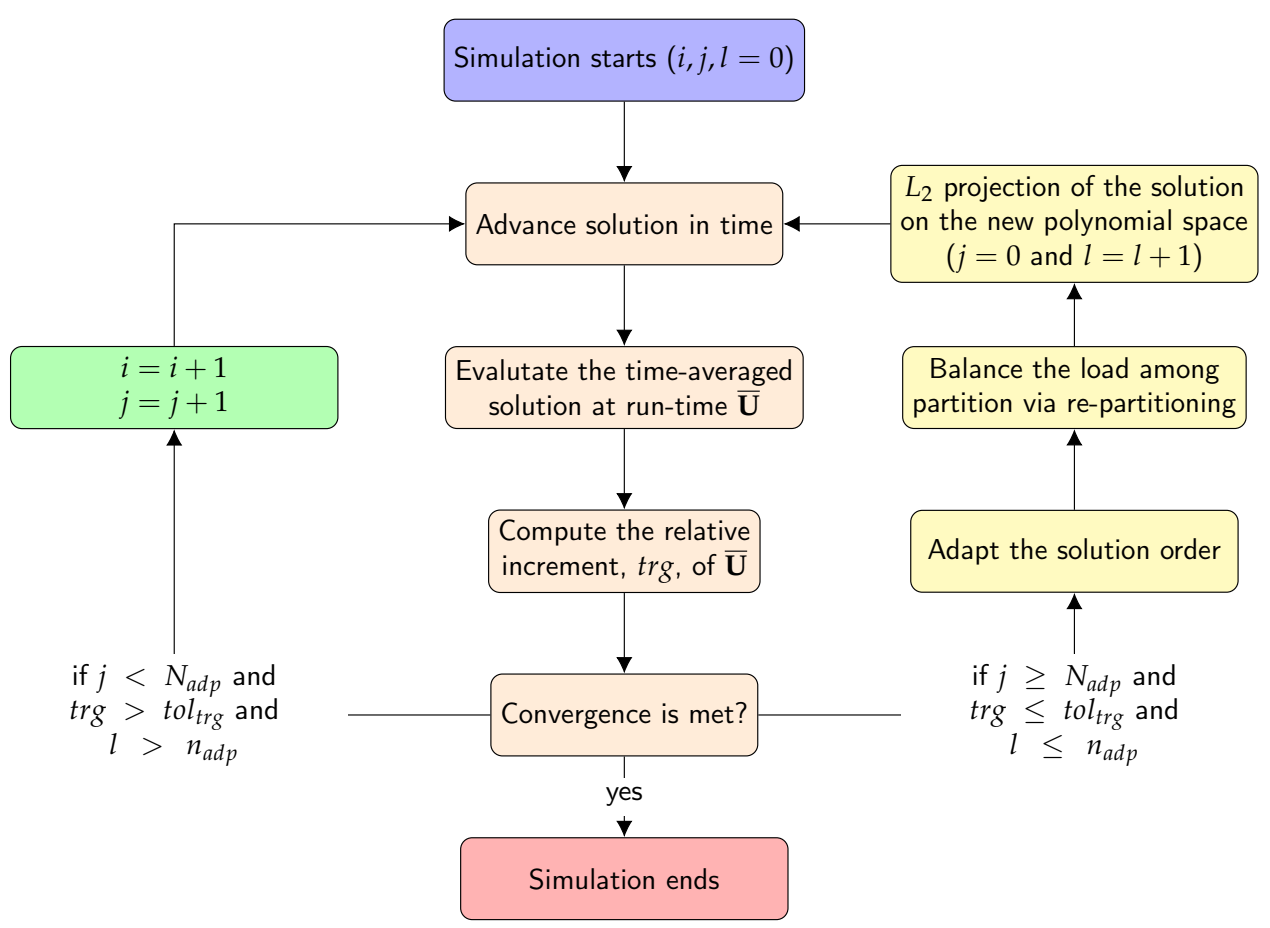

Figure 2. Flowchart of the $p$-adaptation strategy.

In this work, the generation of the curved high-order meshes, suitable for the DG method, is performed using the GridPro [40] software. Different structured meshes with high-order hexahedral elements are generated for each geometry. The software elevates the linear meshes into 2nd- (quadratic edges), 3rd- (cubic edges) or 4th- (quartic edges) order meshes. A cubic representation of the edges is adopted for the simulations presented in the following as a good trade-off between accuracy and computing time.

The "original" DrivAer fastback model [11] has been slightly simplified, removing the handles and the rear-view mirrors, smoothing the underbody and closing the front radiator grid. Figure 3 shows the geometry of the car body used for the simulations in Section 3.2, while Figures 4 and 5 show the corresponding high-order mesh. The mesh consists of 406,304 cubic-edges hexahedral elements.

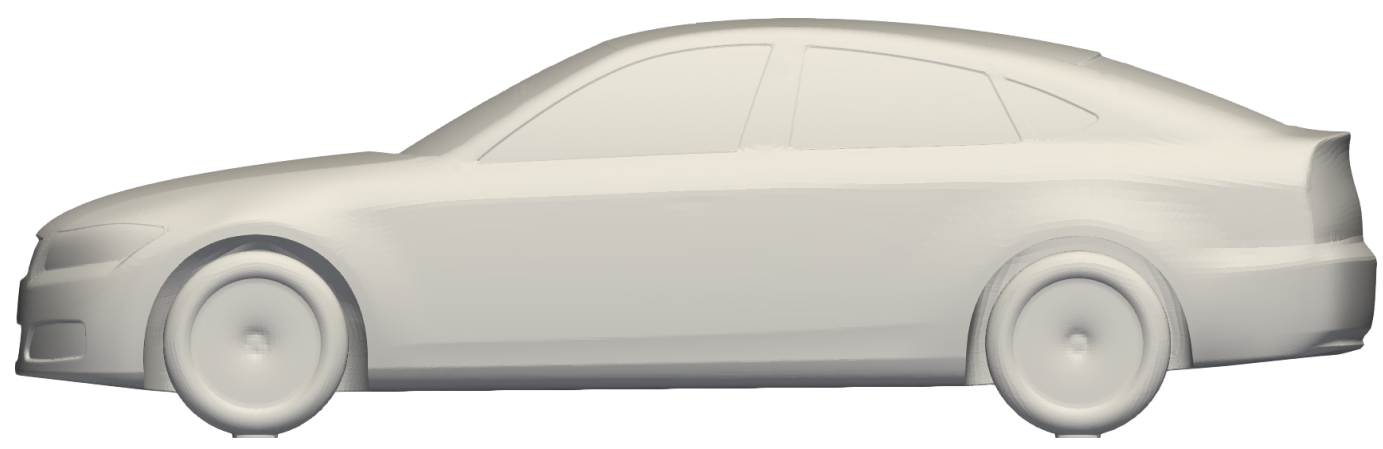

Figure 3. DrivAer car model adopted for the simulations presented in this work. 


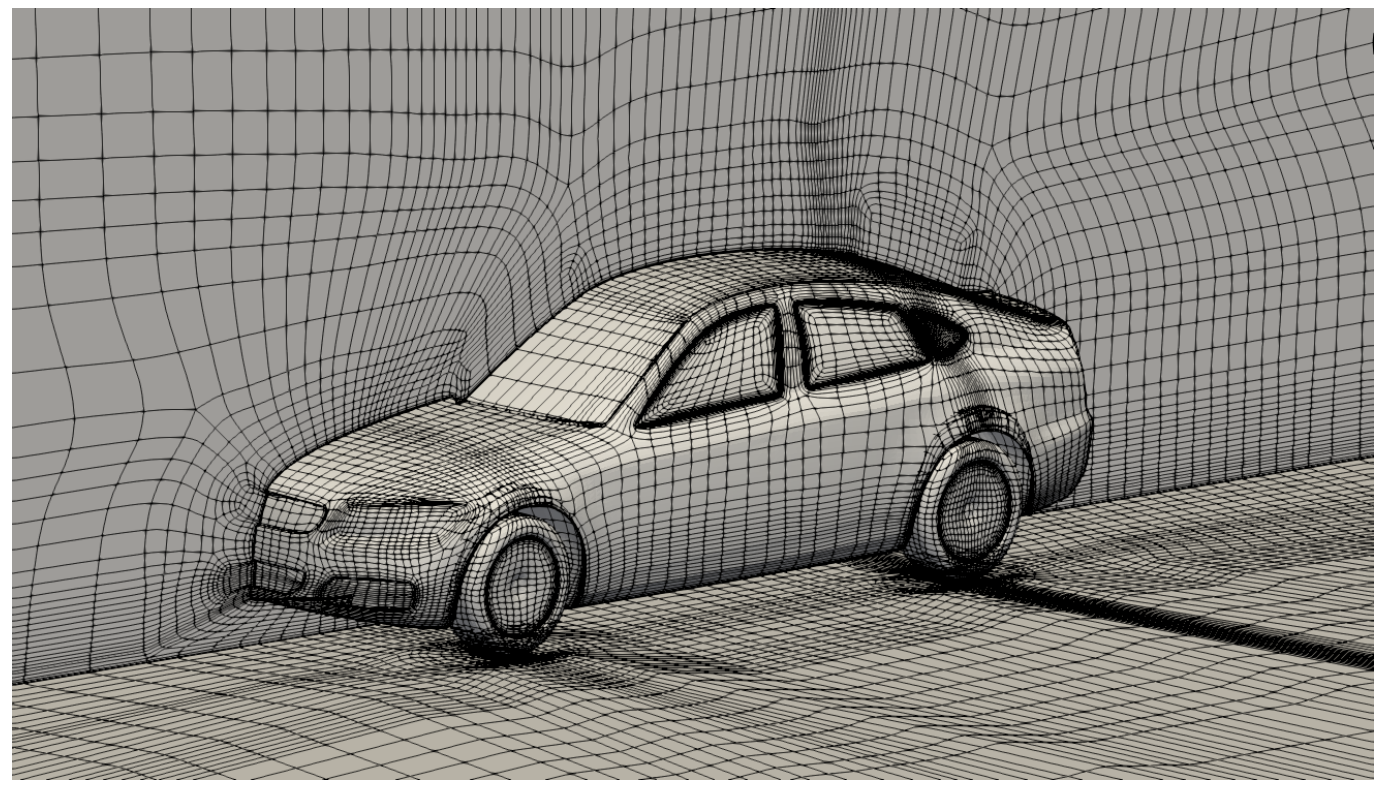

Figure 4. DrivAer model-Mesh with 406304 cubic-edges hexahedral elements.
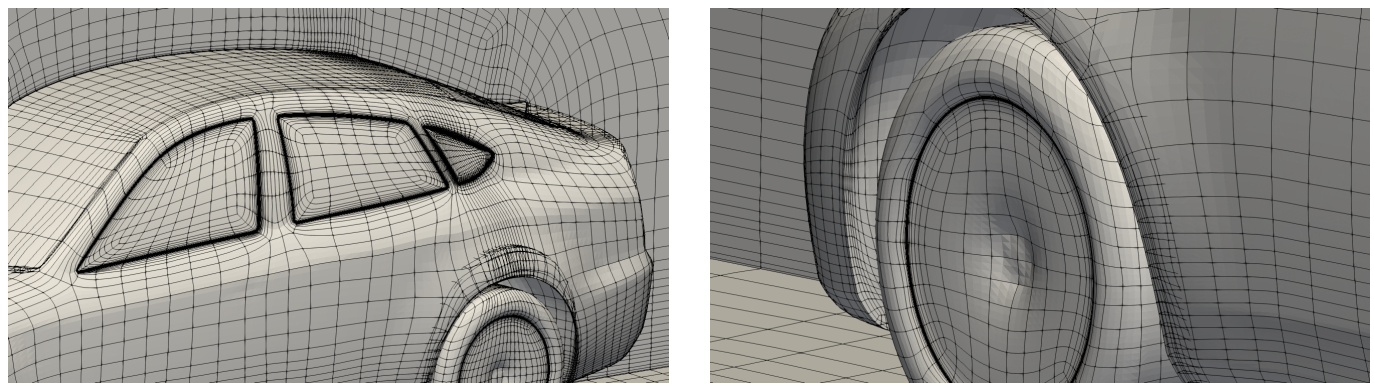

Figure 5. DrivAer model-Detail of the mesh near the rear end of the body (left) and the front wheel (right).

\section{Results}

In this section the results of the ILES for the following geometries are presented: (i) a simplified DrivAer fastback model to asses the influence of the Reynolds number and the absence of the wheels on the aerodynamic performance; (ii) the DrivAer fastback model to assess the capability of the proposed solver to deliver an accurate solution with a moderate number of DoFs. The accuracy of the results are assessed comparing the pressure and aerodynamic coefficients and the flow fields with experimental data [11] and reference CFD solutions $[6,11,13,41]$. The pressure coefficient is defined as

$$
c_{P}=\frac{2\left(p-p_{\infty}\right)}{\rho U_{\infty}^{2}},
$$

where $p_{\infty}$ and $U_{\infty}$ are the pressure and velocity at free stream conditions, $\rho$ the constant density and $p$ the pressure. The drag and lift coefficients are defined as

$$
\begin{aligned}
& c_{D}=\frac{2 F_{x}}{\rho U_{\infty}^{2} A_{r e f}}, \\
& c_{L}=\frac{2 F_{z}}{\rho U_{\infty}^{2} A_{r e f}},
\end{aligned}
$$

where $F_{x}$ and $F_{z}$ are the forces acting on the vehicle along the $x$ (streamwise) and $z$ (normal to the ground) directions, and $A_{r e f}$ is the vehicle frontal area. 
The simulations are performed on a Linux cluster at the University of Bergamo (AMD EPYC 7501 at $2.0 \mathrm{GHz}$ ) and on the MARCONI A-2 cluster at CINECA (Intel KNL at $1.4 \mathrm{GHz})$.

\subsection{Flow around a Simplified DrivAer Model}

The simplified DrivAer model is considered to estimate the effect of the Reynolds number and of the absence of the wheels on the aerodynamics performance. Two different Reynolds number have been considered, $R e_{L}=4.87 \times 10^{6}[6,11,13,41]$ (high-Reynolds number case) and $R e_{L}=2.435 \times 10^{6}$ (low-Reynolds number case), based on the model length $L=4.6 \mathrm{~m}$ and the freestream conditions $\left(u_{\infty}=8 \mathrm{~m} / \mathrm{s}\right.$ and $u_{\infty}=16 \mathrm{~m} / \mathrm{s}$ for the lower and higher Reynolds number, respectively).

The wheelhouses are closed to compare the predicted results with the complete model investigated in Section 3.2 and the data available in literature. Experimental data [11] are available only for $R e_{L}=4.87 \times 10^{6}$ with a freestream turbulence intensity $T u_{\infty}<0.005$. The simulations presented in the following have been performed considering a laminar inlet.

The domain is a box of dimension $10.87 \mathrm{~L} \times 2.17 \mathrm{~L} \times 2.43 \mathrm{~L}$, where $x$ is the streamwise direction, $y$ the spanwise direction and $z$ the direction normal to the ground. The inlet section is at $2.67 \mathrm{~L}$ from the car. A no-slip boundary conditions is applied to the model, while the ground moves at the same velocity as the free stream. According to [13], a symmetric halfbody configuration has been considered for the simulations, and the symmetry condition is prescribed on the $y=0$ symmetry plane. All the simulations are performed with a very coarse mesh, consisting of 113,780 cubic-edges hexahedral elements. Figures 6 and 7 show the mesh with details near the side pillars and the rear end of the model. The averaging time is $\sim T_{C}$ for the low-Reynolds number case and $\sim 3.2 T_{C}$ for the high-Reynolds number case, where $T_{C}$ is a convective time computed according to the freestream velocity and the length of the model. The time step is set equal to $\Delta t=f T_{C}$, where $f=1 \times 10^{-3}$. The maximum polynomial degree of the solution is $\mathbb{P}^{2}$.

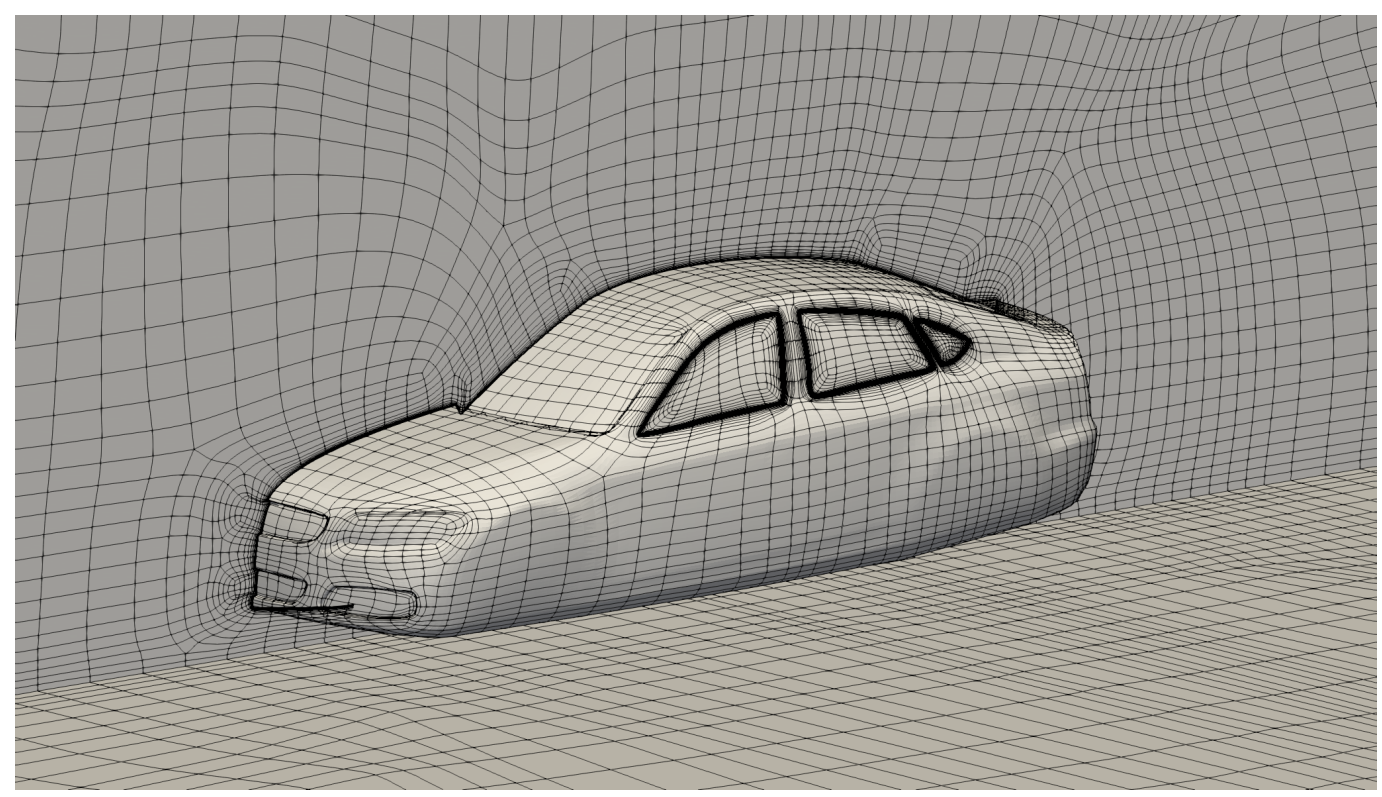

Figure 6. Simplified DrivAer model—Surface mesh of the DrivAer model, 113,780 cubic-edges hexahedral elements. 

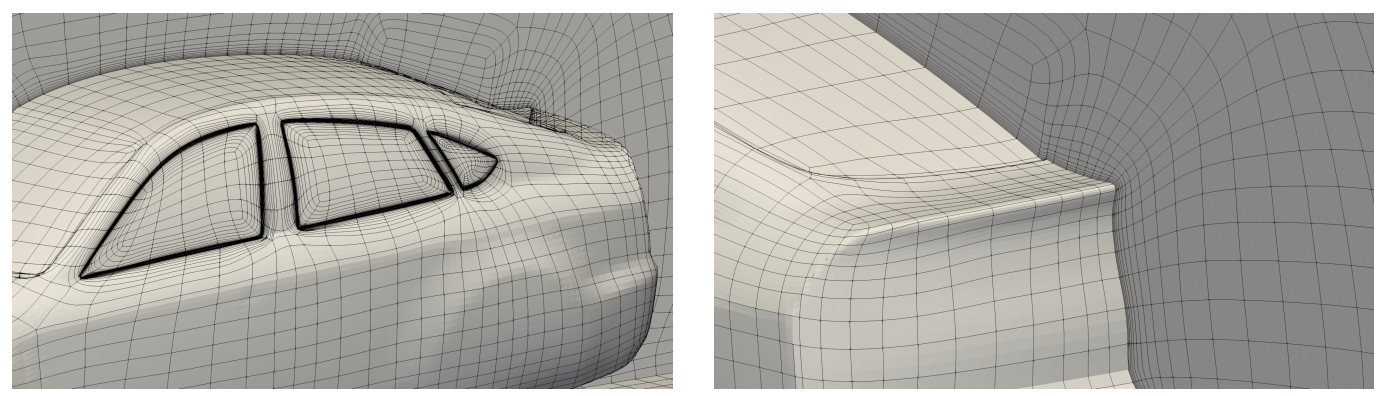

Figure 7. Simplified DrivAer model—Detail of the mesh near the side pillars (left) and the rear part of the body (right).

Figure 8 shows the polynomial degree distribution $\mathbb{P}^{n}$ of the solution, where the final number of DoFs per equation is 591,656 and 601,544 for the low- and high-Reynolds number case, respectively. The front part of the model, the cowl top, the windshield, the A-pillar and the rear part of the model are the regions with higher order elements. Figure 8 shows only the order distribution on the model surface, but high order elements are present also in the wake region. The polynomial degree distribution of the solution after the adaptation process is very similar between the low- and high-Reynolds number cases.
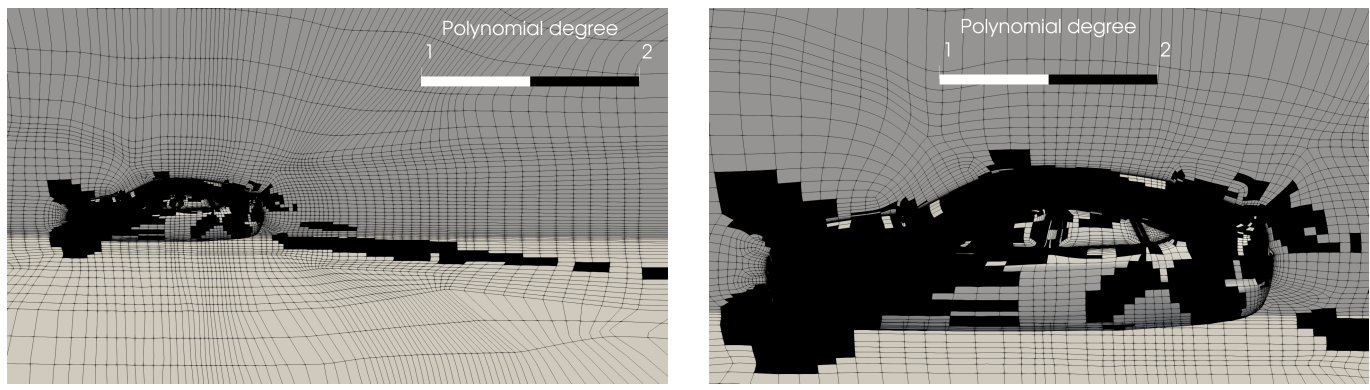

Figure 8. Simplified DrivAer model-Polynomial degree distribution $\mathbb{P}^{n}$ of the solution in the low-Reynolds number case far from the model (left) and near to the model (right).

Figure 9 shows the pressure coefficient, $c_{P}$, distribution on the symmetry plane for the body (left) and underbody (right) of the car for the low Reynolds number case. The predicted results are in good agreement with the experimental results [11] and the LES results [13] (all references are for the $R e_{L}=4.87 \times 10^{6}$ and full model case). After the stagnation point on the front part of the model, flow accelerates and the pressure decreases. The minimum value is lower in the simulation. A first separation appears at the junction between the bonnet and the windshield $(x / L \sim 0.23)$, while a second one appears at the cowl of the windshield $(x / L \sim 0.3)$. The pressure decreases as the flow accelerates over the windshield. The flow passes on the roof, and the pressure slightly increases, while in the experiment it is almost constant. The different behaviour of the $c_{P}$ on the roof could be ascribed to the support that holds the model in the wind tunnel during the measurements. The pressure rises more steeply on the rear window. The rear part of the model, after the end of the rear window, shows widespread results. However, high-fidelity simulations, i.e., reference LES and the proposed ILES simulations, share a similar trend.

On the underbody (see left side of Figure 9) the flow separates after the front grid of the radiator and reattaches near the front wheels. The region between the wheelhouses is characterized by an almost constant $c_{P}$. The smooth underbody behaves like a diffuser, generating a downforce effect.

Figure 10 show the $c_{P}$ distribution on the plane $z=0.06$ for the low-Reynolds number case. The $c_{P}$ distribution shows some discrepancies with experimental data, motivated by the absence of the front rotating wheels, which alter the flowfield near the doors region. The rear part seems to be similar to the experimental data, suggesting a lower influence 
of the rear wheels, but the flow separation at the end of the model is more pronounced. Figures 11 and 12 show the pressure coefficient distributions for the high-Reynolds number case at the same sections described above $(y=0$ and $z=0.06)$. In addition, in this case the $c_{P}$ distribution is in good agreement with references, i.e., the experimental data of Heft et al. [11] and the LES results Rüttgers et al. [13].

An in depth comparison between low and high Reynolds results shows some differences. In particular, Figure 9 (left) shows at $x / L \simeq 0.15$ a pressure drop that corresponds to a laminar separation bubble. This difference can be explained by the lower Reynolds number, i.e., by the lower velocity approaching the car model. In fact, in the hood region, characterised by an adverse pressure gradient, the lower velocity of the flow promotes the onset of a laminar separation bubble. As expected, the predicted results for the higher Reynolds number on the symmetry plane for the body (see left side of Figure 11) are very similar to the reference data and demonstrate that the wheels do not influence significantly the pressure distribution. On the contrary, the results for the underbody (see right side of Figure 11) still show the detachment after the radiator grid and an underestimation of the $c_{P}$ in the doors region. However, starting from the rear wheels, the $c_{P}$ distribution is almost coincident with the experimental data, suggesting a lower influence of the rear wheels on the predicted flow field.

Finally, on the plane $z=0.06$ (see Figure 12) the discrepancy in the doors region is still evident, confirming also in this case the importance of considering the frontal wheels to preserve the simulation accuracy. After the rear wheel, the pressure increase is correctly predicted, but as for the low-Reynolds case, the final separation is larger.
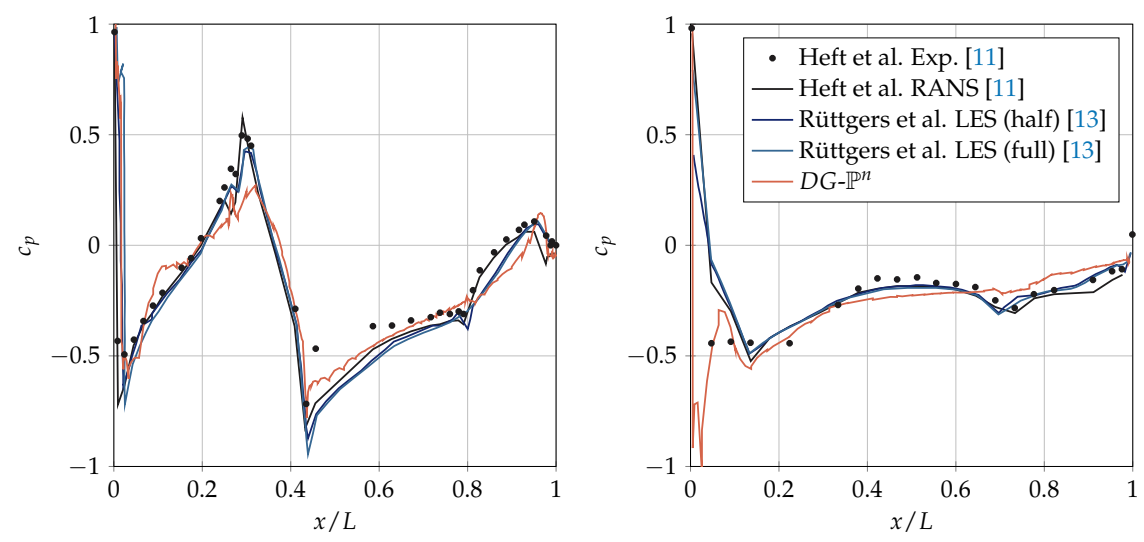

Figure 9. Simplified DrivAer model-Time-averaged pressure coefficient distribution on the symmetry plane for the body (left) and underbody (right) of the car, low-Reynolds number.

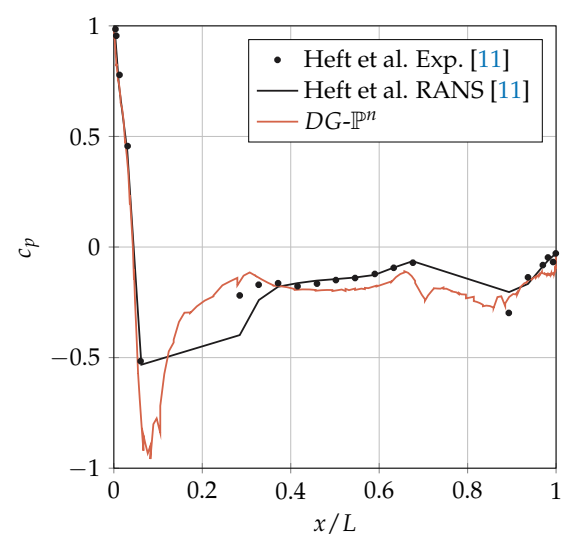

Figure 10. Simplified DrivAer model-Time-averaged pressure coefficient distribution on the plane $z=0.06$, low-Reynolds number. 

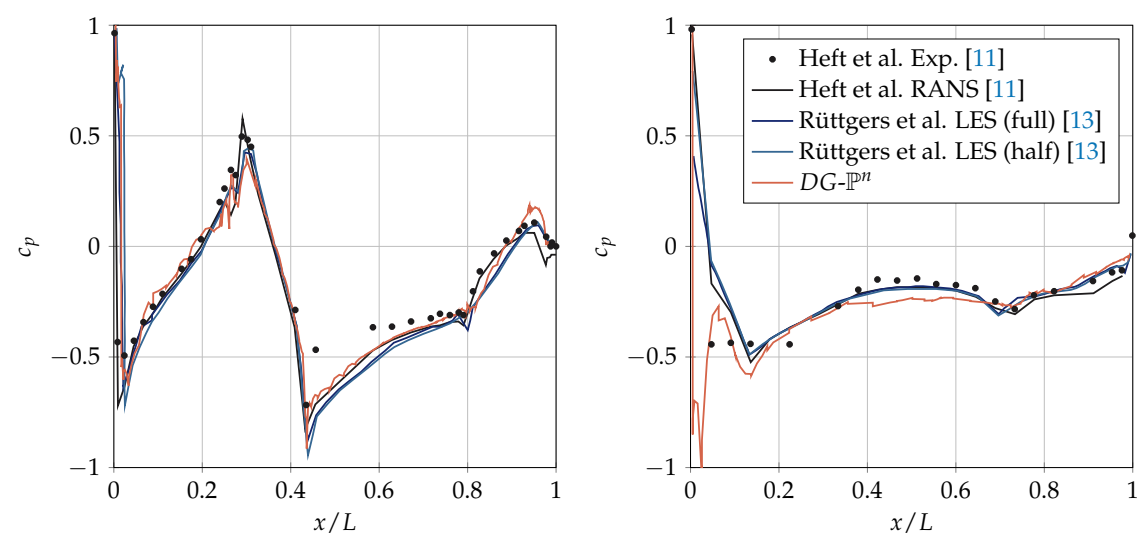

Figure 11. Simplified DrivAer model-Time-averaged pressure coefficient distribution on the symmetry plane for the body (left) and underbody (right) of the car, high-Reynolds number.

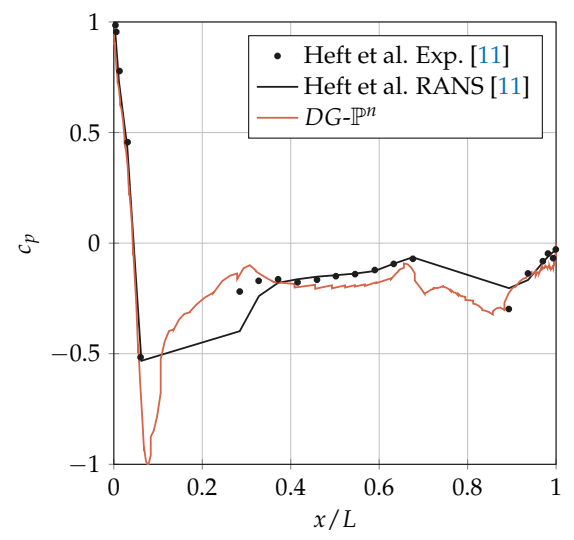

Figure 12. Simplified DrivAer model-Time-averaged pressure coefficient distribution on the plane $z=0.06$, high-Reynolds number

Figure 13 show the time-averaged pressure contours (top-left), the time-averaged pressure coefficient distribution on the windshield (top-right), the front side window (bottomleft) and the back window (bottom-right) for the low-Reynolds number case. The views and the scales of the pressure coefficient contours are the same used by Heft et al. [11] to ease the comparison.

It can be observed that the pressure coefficient distribution on the windshield and back window are similar to the RANS simulations by Heft et al. [11], while some discrepancies are evident in the zone near the pillars. The side window shows a more pronounced low pressure region, probably due to a not correct prediciton of the A-pillar vortices. As demonstrated in Section 3.2, this difference can be ascribed to the absence of the wheels.

The predicted drag and lift coefficients for the low- and high-Reynolds number cases are summarised in Table 1 and compared with experimental and numerical results of Heft et al. [11]. The negative value of $c_{L}$ confirms that the underbody acts like a diffuser. The $c_{D}$ slightly changes increasing the Reynolds number, confirming the experimental findings [11]. On the contrary, the $c_{L}$ shows a huge variation, which can be ascribed to the different distribution of the pressure on the roof of the car. The $c_{D}$ is slightly overpredicted and confirms the trend summarised in Table 2, where some numerical references, e.g., Aljure et al. [12], Ashton et al. [6] and Guilmineau et al. [4], predict a drag coefficient larger than 2.5 .

\subsection{Flow around the DrivAer Model}

The turbulent flow around the DrivAer model is presented in this section for a Reynolds number $\operatorname{Re}_{L}=4.87 \times 10^{6}[6,11,13,41]$, based on the model length $L=4.6 \mathrm{~m}$ 
and the freestream conditions. The rear mirrors are not considered and the underbody is smooth.
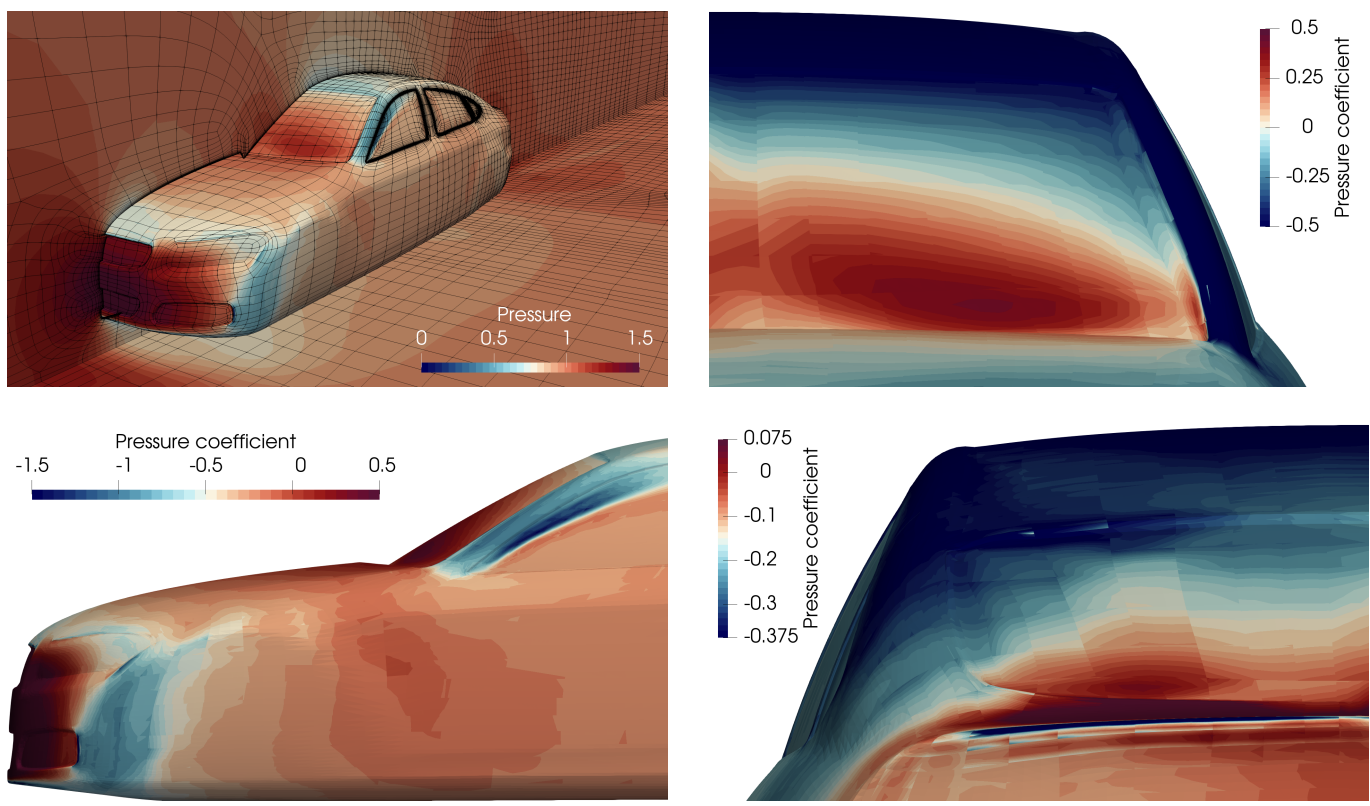

Figure 13. Simplified DrivAer model-Time-averaged pressure contours (top-left) and timeaveraged pressure coefficient distribution on the windscreen (top-right), the front side window (bottom-left) and the back window (bottom-right), low-Reynolds number.

Table 1. Simplified DrivAer model—Predicted drag, $c_{D}$, and downforce, $c_{L}$, coefficients for low- and high-Reynolds number case. Results are compared with reference data.

\begin{tabular}{lccc}
\hline & Method & $c_{D}$ & $c_{L}$ \\
\hline \multirow{2}{*}{ Heft et al. [11] } & RANS $k$ - $\omega$ SST & 0.124 & - \\
& Experiment & 0.125 & - \\
\hline$D G-\mathbb{P}^{n}$ (low Re) & ILES & 0.1561 & -0.1352 \\
\hline$D G-\mathbb{P}^{n}$ (high Re) & ILES & 0.1605 & -0.0753 \\
\hline
\end{tabular}

The domain is a box of dimension $10.87 \mathrm{~L} \times 2.17 \mathrm{~L} \times 2.43 \mathrm{~L}$, where $x$ is the streamwise direction, $y$ the spanwise direction and $z$ the direction normal to the ground. The inlet section is at $2.67 \mathrm{~L}$ from the car.

A no-slip boundary condition is applied to the model, the wheels rotate with a prescribed angular velocity and the ground moves at the same velocity of the free stream. Computations with a symmetric half-body configuration are performed, and the symmetry condition is prescribed to the $y=0$ plane. All the simulations are performed with a very coarse mesh, consisting of 406,304 cubic-edges hexahedral elements. Figures 4 and 5 show the computational domain and some details near the rear end and the front wheel. Notice the small amount of elements used to discretize the car, especially if compared to the references. In fact, Rüttgers et al. [13] carried out LES on the full and symmetric half-body geometry with meshes of 24,80 and $130 \times 10^{6}$ elements for the full geometry and $65 \times 10^{6}$ elements for the symmetric half-body geometry. Heft et al. [11] and Frank et al. [41] carried out Reynolds-averaged Navier-Stokes (RANS) simulations, using $k-\omega$ SST and $k-\omega$ SAS SST models on a mesh made of $19.4 \times 10^{6}$ and $110 \times 10^{6}$ elements, respectively. Aljure et al. [12] carried out WMLES on the full geometry with meshes of 6.5, 53 and $79 \times 10^{6}$ elements. Ashton et al. [6] performed RANS simulations with meshes of 18,37 and $80 \times 10^{6}$ elements, while meshes of 80 and $100 \times 10^{6}$ elements were used for DES-type simulations. 
Averaging time is $\sim T_{C}$, where $T_{C}$ is a convective time computed according to the freestream velocity and the length of the model. The time step has been set to $\Delta t=f T_{C}$, where $f=6 \times 10^{-4}$. The maximum polynomial degree of the solution is $\mathbb{P}^{2}$.

Figure 14 shows the polynomial degree distribution $\mathbb{P}^{n}$ of the solution on the surface of the car model. The front part of the model, the windshield, the A-pillar, the wheels and the rear part of the model require higher order elements. As expected, the comparison of the polynomial degree distribution between the present and the simplified (see Figure 8) DrivAer configuration shows that rotating wheels require high-order elements. The DoFs per equation are 2,112,776.
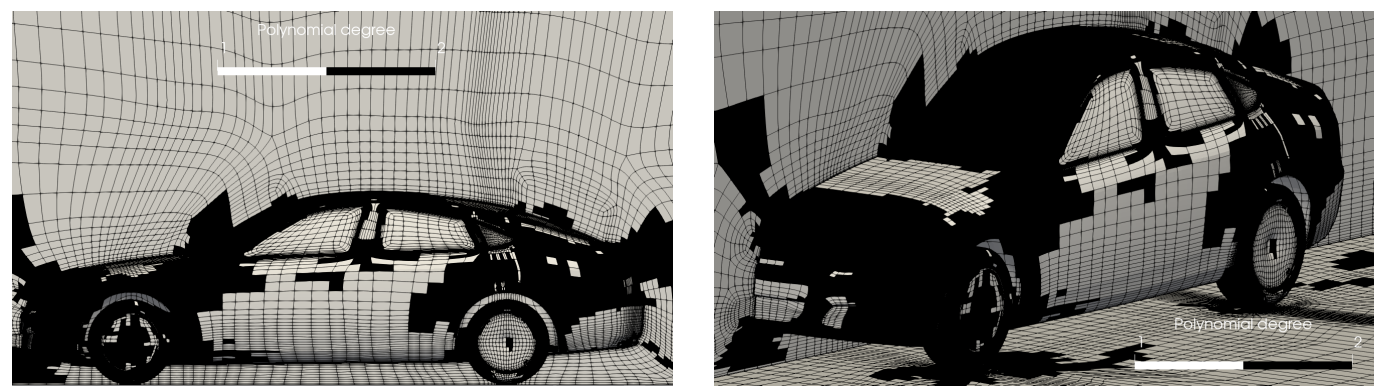

Figure 14. DrivAer model-Polynomial degree distribution $\mathbb{P}^{n}$ of the solution from the lateral (left) and the front (right) point of view.

The pressure coefficient distribution on the symmetry plane $y=0$ shows the same behaviour of the simplified DrivAer model (see Figure 9). Differences are evident for the $c_{P}$ distribution on the plane $z=0.06$ that intersects the wheels, shown in Figure 15 . The flow accelerates from the stagnation point and detaches near the front wheel. In the doors region the pressure recovers slowly. After the second flow separation at the rear wheel, there is a sudden pressure recovery in the rear part of the model. However, the simulation predicts a separation bubble that reattaches just before the end of the model. The predicted distribution is in good agreement with the WMLES simulations of Aljure et al. [12]. The comparison between the predicted results presented in this section and in Section 3.1 (see Figures 12 and 15) puts in evidence the importance of the wheels in the prediction of the side flow field.

Figure 16 shows the time-averaged pressure coefficient contours on the body (top-left), underbody (top-right), the windshield (middle-left), the front side window (middle-right) and the back window (bottom). The views and the scales of the pressure coefficient contours for the windshield, the front side window and the back window are the same used by Heft et al. [11] to ease the comparison. The distribution on the top and bottom view can be compared with the LES results of Rüttgers et al. [13], showing a good agreement. A discrepancy can be observed in the front part of the bottom distribution, where the predicted results show a separation that generates a sudden decrease of the pressure. Moreover, near the A-pillar the low pressure region is less evident in the predicted results due to the absence of the rear mirrors.

The $c_{P}$ contours on the windshield and on the back window are very close to the reference. In the central zone of the back window there is a small low pressure region that is not present in the reference RANS simulations, which suggests the presence of a small detached zone at the junction between the roof and the back window. Moreover, in contrast to the simulation without the wheel, the location of vortex in the A-pillar zone is now in agreement with the references, even if the low pressure region is more widespread for the absence of the rear mirror. 


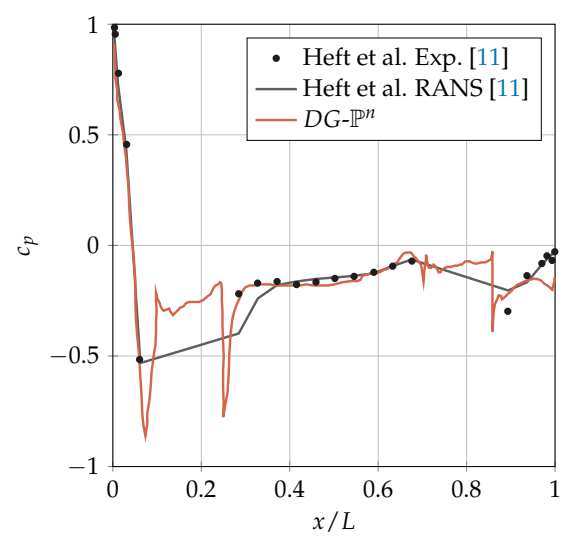

Figure 15. DrivAer model-Time-averaged pressure coefficient distribution on the $z=0.06$ plane.
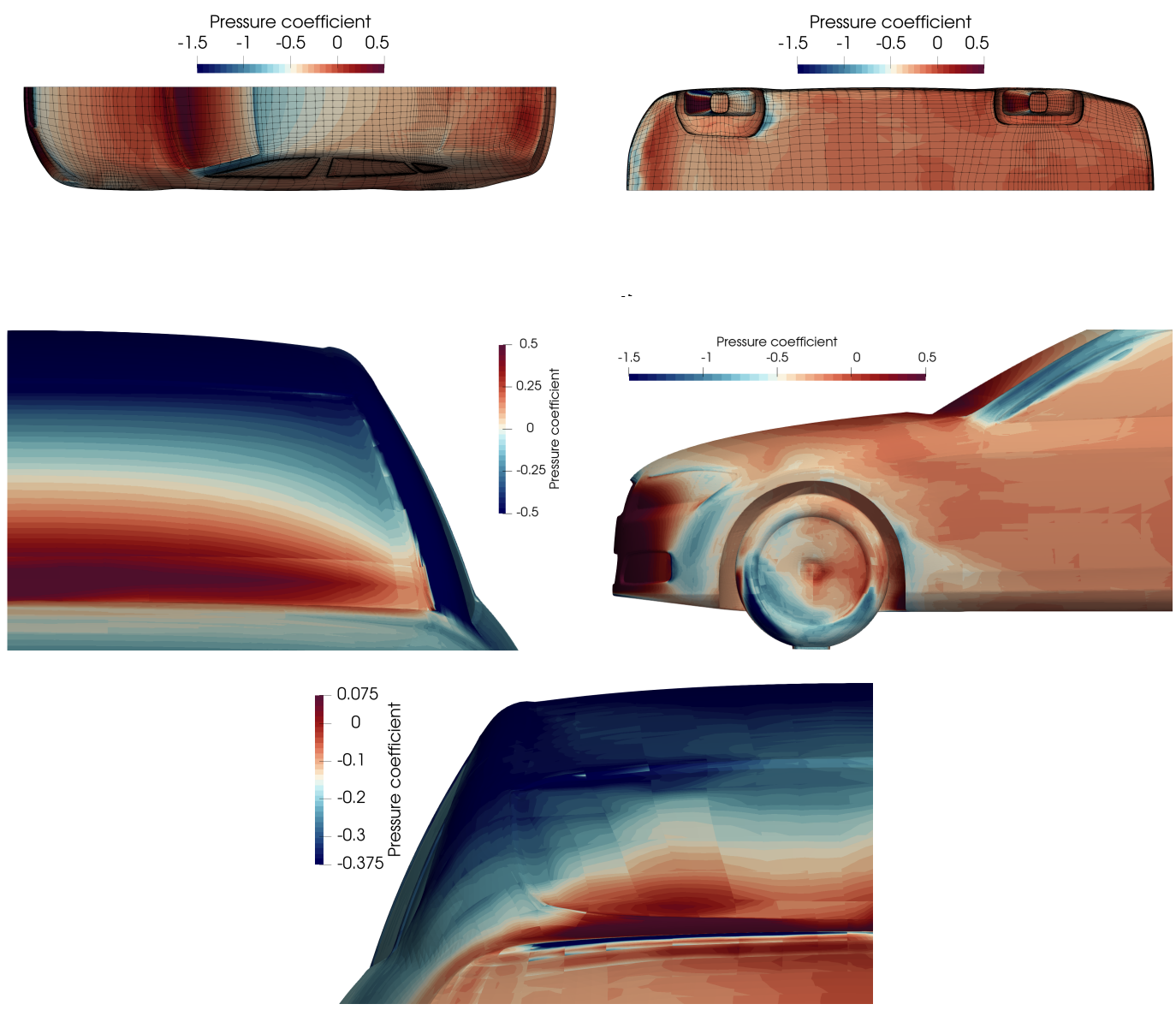

Figure 16. DrivAer model-Time-averaged pressure coefficient distribution on the body (top-left), the underbody (top-right), the windshield (middle-left), the front side window (middle-right) and the back window (bottom).

Figures 17 and 18 show the time-averaged $x$-velocity contours on the slices $x / L=$ $(0.173,0.273,0.423)$ and $x / L=(0.88,0.98,1.10)$, respectively. In particular, the slice at $x / L=0.173$ cuts the wheel, showing the flow field inside the wheel house, which is characterised by a negative $x$-velocity. In fact, the flow inside the wheel house follows the direction prescribed by the wheel angular velocity. Moreover, near the ground contact, two counter rotating vortices generated ahead of the wheel are observed. The slice at $x / L=0.273$ shows that vortical structures created by the wheel are convected downstream, influencing the side flow field. Finally, the slice at $x / L=0.423$ intersects the A-pillar and 
shows the A-pillar vortex that is convected downstream. The slices on the rear part of the model are the same used by Ashton et al. [6]. The contours are in agreement with the IDDES simulations. In particular, the slice at $x / L=0.88$ shows the vortical structures created by the rear wheel and the small separation on the back window. The slices at $x / L=0.98$ and $x / L=1.10$ show the development of the wake. In the lower part there is a region with positive $x$-velocity coming from the underbody that is not yes mixed with the wake.
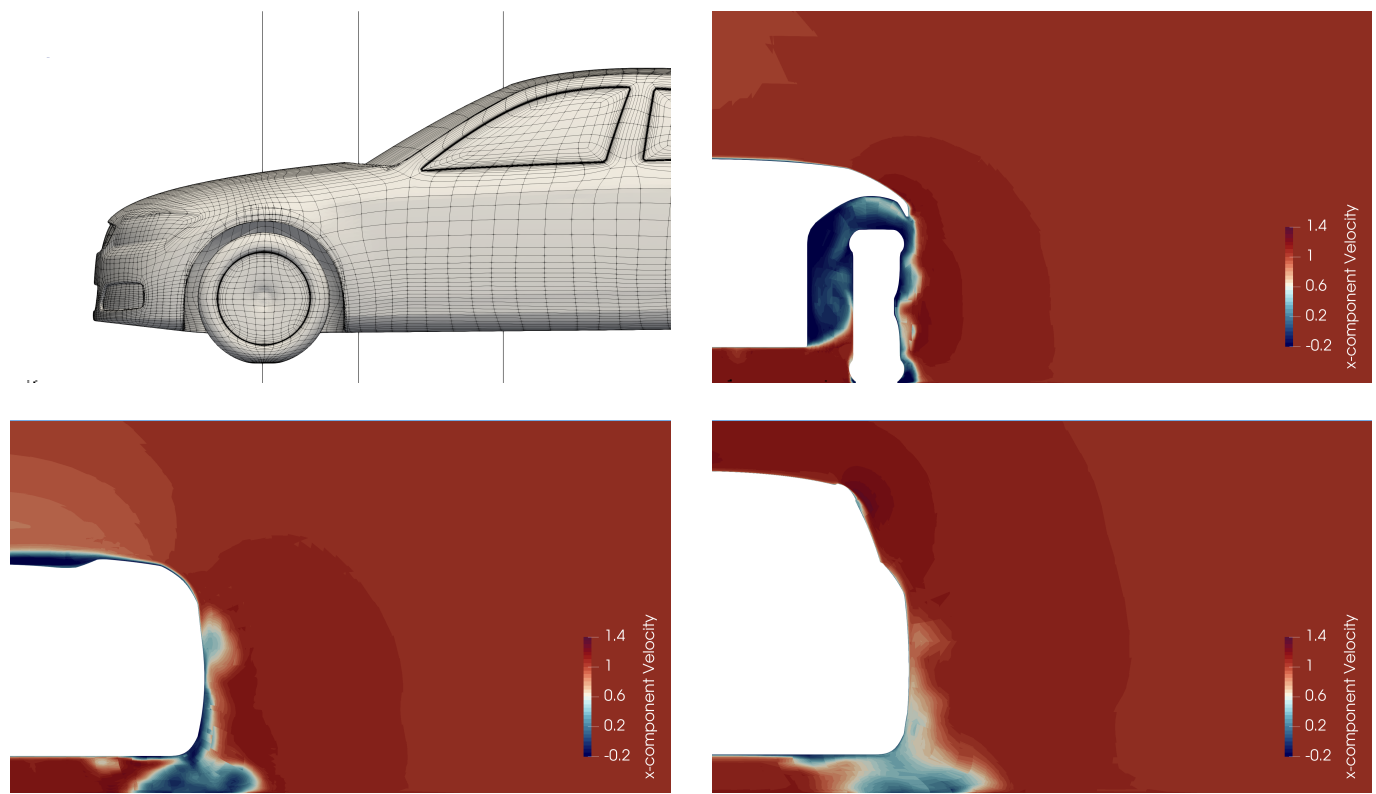

Figure 17. DrivAer model (top,left) -Time-averaged $x$-velocity contours at different slices: $x / L=$ 0.173 (top,right), $x / L=0.273$ (bottom,left), and $x / L=0.423$ (bottom,right).
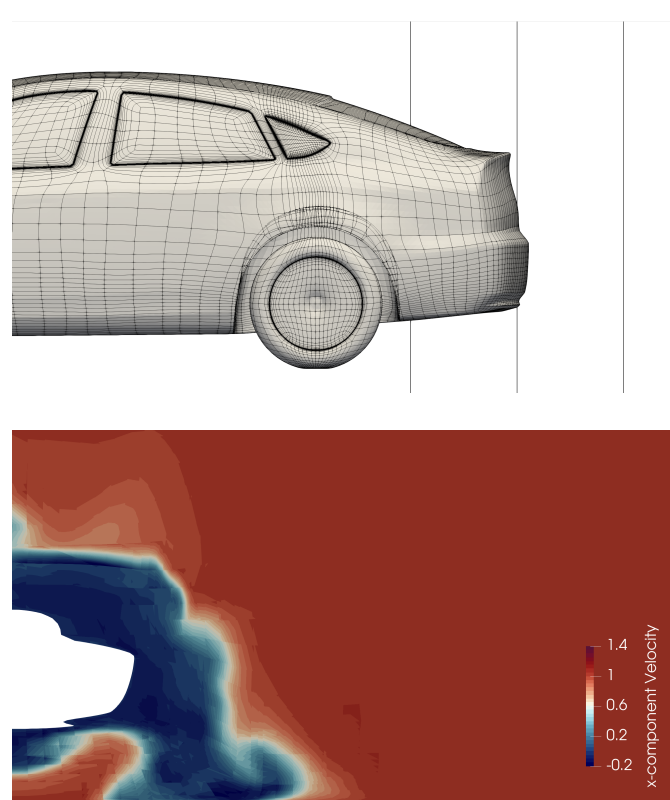
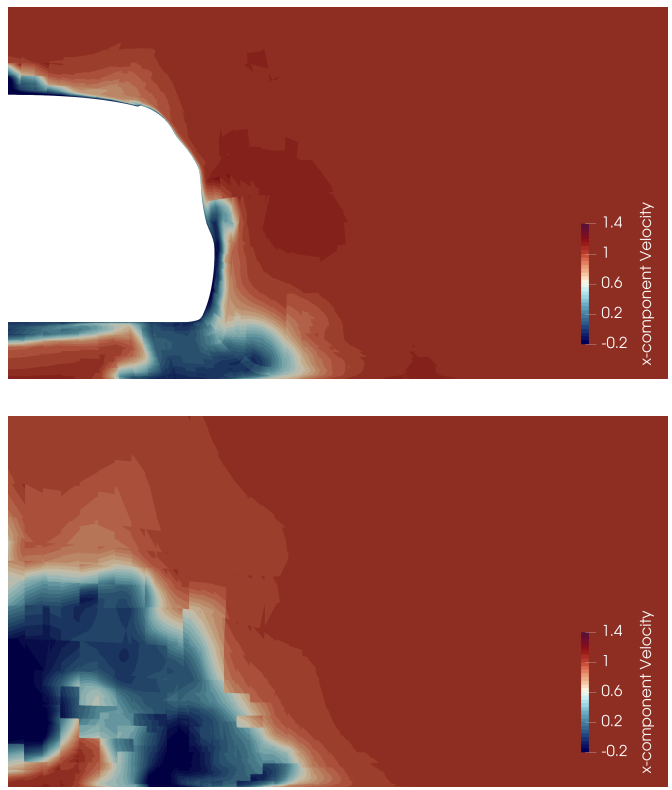

Figure 18. DrivAer model (top,left) - Time-averaged $x$-velocity contours at different slices: $x / L=$ 0.88 (top,right), $x / L=0.98$ (bottom,left), and $x / L=1.10$ (bottom,right).

Figure 19 shows the details of the time-averaged velocity vectors behind the front wheels, in the gap between the cowl top and the windshield and on the rear slant. In the wheel region the horseshoe vortices are evident, which are created just ahead of the wheel 
and are convected downstream. The two counter rotating vortices can be observed also in the LES simulations of Rüttgers et al. [13]. Moreover, the recirculating zone in the gap between the cowl top and the windshield can be observed, while the recirculation on the rear slant can be noticed only near the symmetry plane, as shown in Figure 19 (bottom-left), where the discontinuity between the roof and the rear slant is higher. Moving towards the side, the discontinuity between roof and the rear slant is not enough to promote separation, as shown in Figure 19 (bottom-right).
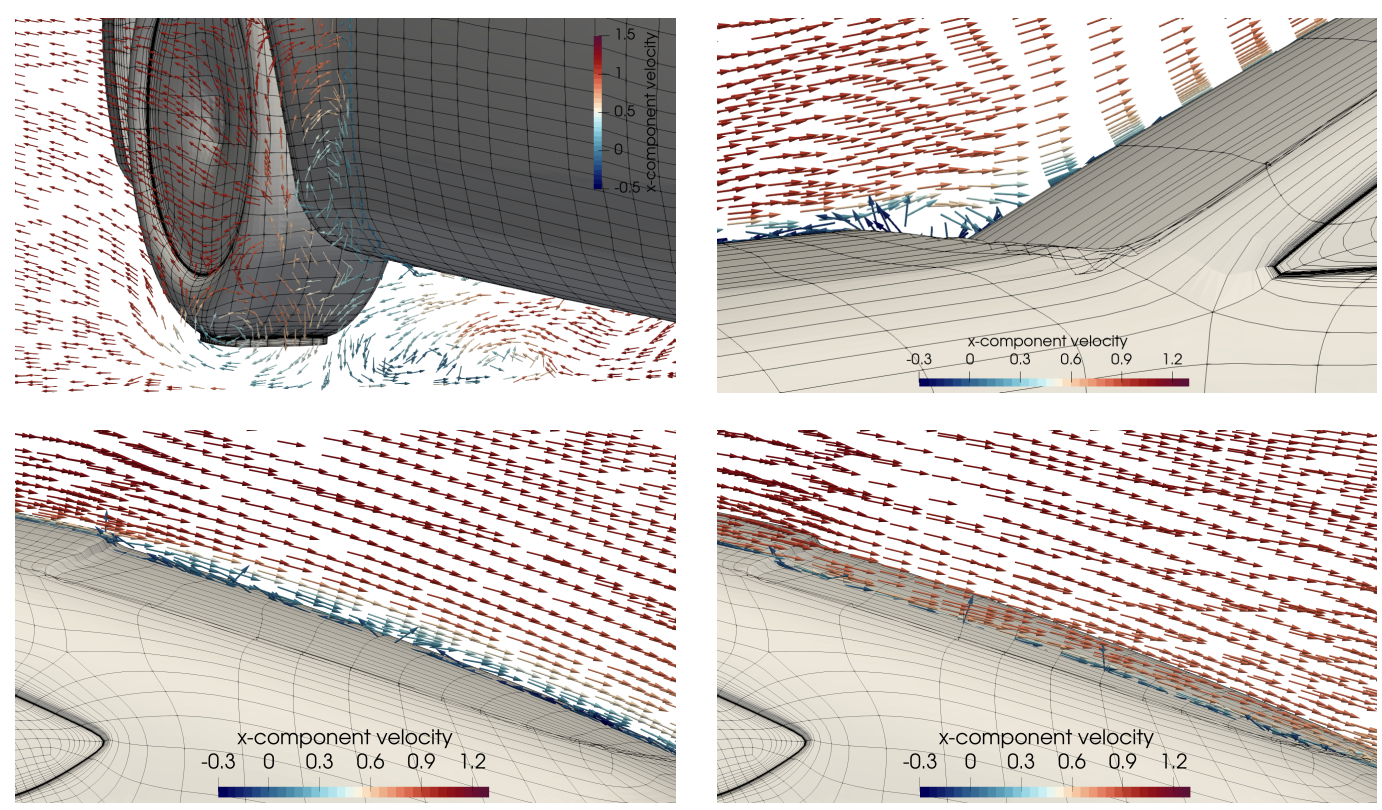

Figure 19. DrivAer model-Details of the time-averaged velocity vectors behind the front wheels (top,left), in the gap between the cowl top and the windshield (top,right) and on the rear slant (bottom).

The predicted drag coefficient is $c_{D}=c_{D \text {, wheel }}+c_{D, \text { body }}=0.2556$, where $c_{D \text {, wheel }}=0.0327$ and $c_{D, b o d y}=0.2229$ are the wheels and the body contributions to the drag, respectively. The drag can be decomposed in the skin friction, $c_{D, v}=0.02 c_{D}=0.0048$, and pressure, $c_{D, P}=0.98 c_{D}=0.2505$ contributions. The results are in good agreement with the reference data, as reported in Table 2. In particular, the predicted $c_{D}$ value overestimates the experimental data, but this behaviour is confirmed also by most numerical references, e.g., Aljure et al. [12], Ashton et al. [6] and Guilmineau et al. [4], which predict a drag coefficient larger than 2.5. As expected, the presence of the wheels heavily influences the aerodynamic performance of the car model. In fact, the down force predicted without the wheel becomes a lift force. The predicted lift coefficient is $c_{L}=c_{L, \text { wheel }}+c_{L, \text { body }}=0.0345$, where $c_{L, \text { wheel }}=-0.1560$ and $c_{L, \text { body }}=0.1912$ are the wheels and the body contributions to the lift, respectively. The lift can be decomposed again in the skin friction, $c_{L, v}=0.0011$, and pressure, $c_{L, P}=0.0334$, contributions.

Figure 20 shows the A-pillar vortex (the streamlines are coloured with the $x$-component velocity for the time-averaged solution), which develops close to the root of the A-pillar and detaches before the roof. The vortex is convected downstream and merges with the C-pillar vortex.

Figures 21 and 22 compare the time-averaged streamlines coloured with the timeaveraged $x$-component velocity contours obtained with the present and the simplified car model. In both cases the flow accelerates from the stagnation point in the front bump area and moves toward the bonnet and the wheel region. Along the sides of the bonnet, a high pressure region (see Figure 16) redirects the flow downward. Moreover, the high pressure zone on the windshield pushes the flow towards the A-pillar. The vortex created at the Apillar root is convected downstream and merges into the wake. The presence of the wheels 
modifies the side flow field: (i) vortices are created at the wheelhouses discontinuities and convected downstream where they merge into the wake; (ii) the front-wheel influences the A-pillar region, as it modifies the direction of the A-pillar vortex (the low pressure region on the side window visible in Figure 13 disappears).

Table 2. DrivAer model - Drag, $c_{D}$, and downforce, $c_{L}$, coefficients in comparison with other reference numerical results and experimental data. DoFs are the degrees of freedom, i.e., the unknown solutions. All the reference solvers are based on cell-centered finite-volume methods, i.e., the DOFs are equal to the mesh elements. DoFs of the simulations for half model are reported projected to the full model simulation in order to have an easy comparison.

\begin{tabular}{lccccc}
\hline & Method & Elements & DoFs & $c_{D}$ & $c_{L}$ \\
\hline & LES & $24 \times 10^{6}$ (full) & $24 \times 10^{6}$ & 0.244 & 0.005 \\
\multirow{4}{*}{ Rüttgers et al. [13] } & LES & $80 \times 10^{6}$ (full) & $80 \times 10^{6}$ & 0.234 & 0.005 \\
& LES & $130 \times 10^{6}$ (full) & $130 \times 10^{6}$ & 0.228 & 0.005 \\
& LES & $55 \times 10^{6}$ (half) & $110 \times 10^{6}$ & 0.274 & 0.005 \\
& LES & $65 \times 10^{6}$ (half) & $130 \times 10^{6}$ & 0.231 & 0.005 \\
\hline \multirow{4}{*}{ Aljure et al. [12] } & WMLES & $9 \times 10^{5}$ (full) & $9 \times 10^{5}$ & 0.293 & 0.036 \\
& WMLES & $6.5 \times 10^{6}$ (full) & $6.5 \times 10^{6}$ & 0.253 & 0.057 \\
& WMLES & $53 \times 10^{6}$ (full) & $53 \times 10^{6}$ & 0.246 & 0.083 \\
& WMLES & $79 \times 10^{6}$ (full) & $79 \times 10^{6}$ & 0.251 & 0.069 \\
\hline \multirow{4}{*}{ Ashton et al. [6] } & RANS SA & $80 \times 10^{6}$ (full) & $80 \times 10^{6}$ & 0.260 & 0.136 \\
& RANS $k-\epsilon$ & $80 \times 10^{6}$ (full) & $80 \times 10^{6}$ & 0.244 & 0.085 \\
& RANS $k-\omega$ SST & $80 \times 10^{6}$ (full) & $80 \times 10^{6}$ & 0.260 & 0.124 \\
& RANS $k$ - B-EVM & $80 \times 10^{6}$ (full) & $80 \times 10^{6}$ & 0.243 & 0.116 \\
& RANS EB-ESM & $80 \times 10^{6}$ (full) & $80 \times 10^{6}$ & 0.248 & 0.075 \\
& SST IDDES & $80 \times 10^{6}$ (full) & $80 \times 10^{6}$ & 0.268 & 0.011 \\
\multirow{2}{*}{ Guilmineau et al. [4] } & SST IDDES & $100 \times 10^{6}$ (full) & $100 \times 10^{6}$ & 0.261 & 0.024 \\
\hline \multirow{2}{*}{ Heft et al. [11] } & RANS EARSM & $38.6 \times 10^{6}$ (full) & $38.6 \times 10^{6}$ & 0.254 & 0.079 \\
& SST DES & $38.6 \times 10^{6}$ (full) & $38.6 \times 10^{6}$ & 0.266 & 0.024 \\
\hline$D G-\mathbb{P}^{n}$ & RANS $k-\omega$ SST & $19.8 \times 10^{6}$ (full) & $19.8 \times 10^{6}$ & 0.241 & - \\
& Experiment & - & - & 0.243 & - \\
\hline
\end{tabular}
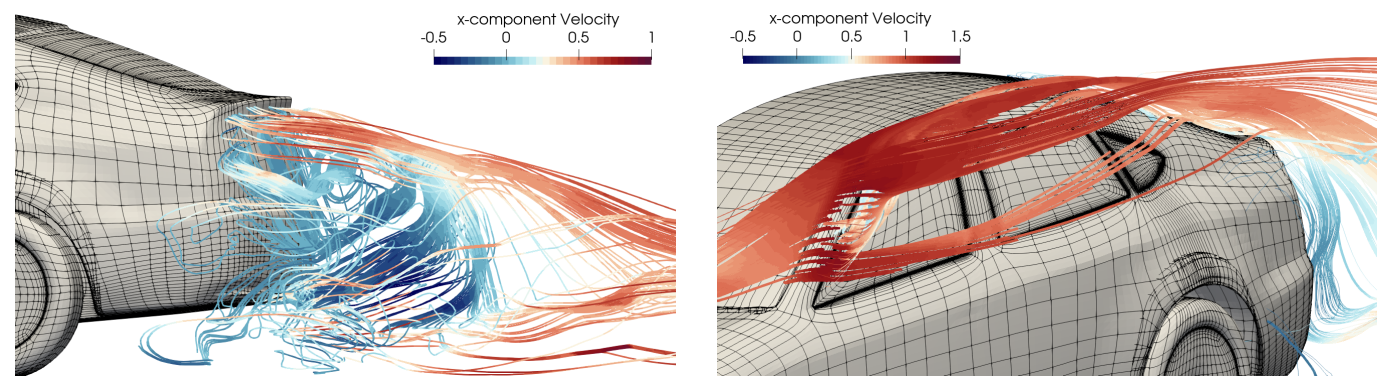

Figure 20. DrivAer model-Details of the streamlines coloured with the $x$-component of the velocity for the time-averaged solution near the rear-end (left) and the A-pillar (right) region. 

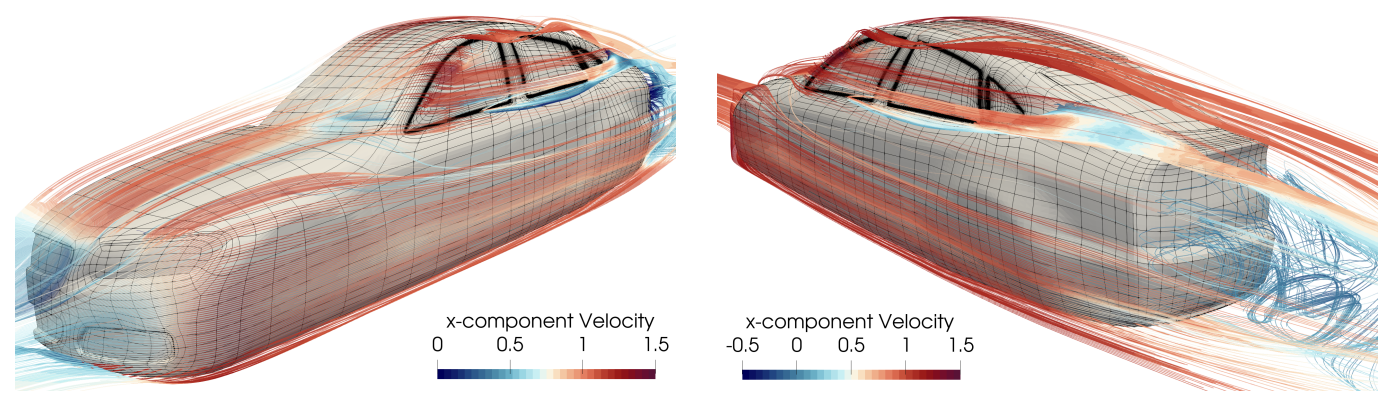

Figure 21. Simplified DrivAer model-Streamlines coloured with the $x$-component of the velocity for the time-averaged solution from the front (left) and the rear (right) point of view.
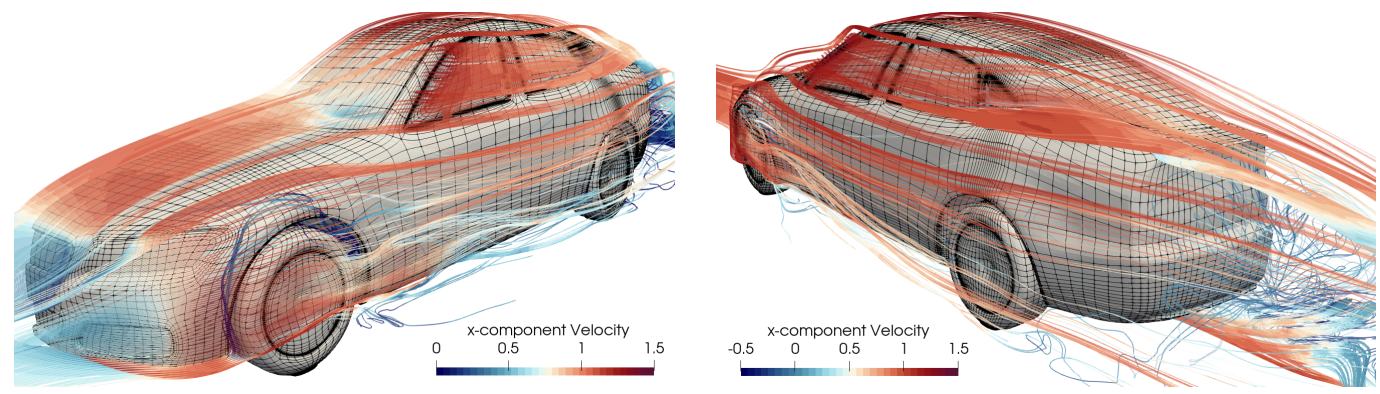

Figure 22. DrivAer model-Streamlines coloured with the $x$-component of the velocity for the time-averaged solution from the front (left) and the rear (right) point of view.

\section{Conclusions}

In the present study simulations have been performed around different geometries of the DrivAer fastback model with an order-adaptive discontinuous Galerkin method.

This work has to be considered as a proof of concept for the use of the present DG solver to capture the main features that characterise the flow around a car, using very coarse high-order meshes and a moderate number of DoFs.

A simplified DrivAer model has been first considered, i.e., without rear mirrors and wheels and with a smooth underbody, to assess the influence of the Reynolds number on the aerodynamic performance. As also shown in the literature, the effect of the Reynolds number (for $R e>2 M$ ) on drag coefficient was found to be negligible, while a considerable difference is observed for the lift coefficient. Moreover, in this configuration the underbody promotes a downforce effect.

The second model also takes into account of the rotating wheels. Our computations have been compared with experimental data and CFD from the literature, showing a good agreement. When including wheels in the model, downforce vanishes due to the different flow evolution on the underbody and vortical structures flow on the car sides due to the discontinuities of the wheelhouses. Moreover, the A-pillar vortex is modified, as the orientation of its rotation axis is changed, generating a different pressure distribution on the pillar itself and the side window.

Ongoing work is devoted to further numerically investigate the DrivAer configuration to corroborate the findings of this paper and to contribute to the creation of a data-base for validation and comparison purposes.

Author Contributions: Conceptualisation, A.C. (Alessandro Colombo); methodology, A.C. (Alessandro Colombo); validation, A.C. (Andrea Crivellini) and A.N.; formal analysis, A.B. and G.N.; investigation, A.B.; resources, A.C. (Alessandro Colombo) and P.C.; data curation, G.N.; writing-original draft preparation, A.G. and G.N.; writing-review and editing, A.C. (Alessandro Colombo) and A.C. (Andrea Crivellini); visualisation, G.N. All authors have read and agreed to the published version of the manuscript.

Funding: This research received no external funding. 
Acknowledgments: The authors would like to thank the Institute of Aerodynamics and Fluid Mechanics at Technical University of Munich for providing the CAD data of the DrivAer models. The authors would like to express their gratitude towards Samuel James and Vimalraj Anbumani from GridPro (Block Structured Mesher) for the support regarding meshing.

Conflicts of Interest: The authors declare no conflict of interest.

\section{References}

1. Hucho, W.; Sovran, G. Aerodynamics of Road Vehicles. Annu. Rev. Fluid Mech. 1993, 25, 485-537. annurev.fl.25.010193.002413. [CrossRef]

2. Kawamata, H.; Kuroda, S.; Tanaka, S.; Oshima, M. Improvement of Practical Electric Consumption by Drag Reducing under Cross Wind. In SAE 2016 World Congress and Exhibition; SAE International: Brooklyn, MI, USA, 2016; doi:10.4271/2016-01-1626. [CrossRef]

3. Rodi, W. Comparison of LES and RANS calculations of the flow around bluff bodies. J. Wind. Eng. Ind. Aerodyn. 1997, 69-71, 55-75. [CrossRef]

4. Guilmineau, E. Numerical Simulations of Flow around a Realistic Generic Car Model. SAE Int. J. Passeng. Cars Mech. Syst. 2014, 7, 646-653. [CrossRef]

5. Ashton, N.; Revell, A. Comparison of RANS and DES Methods for the DrivAer Automotive Body. In SAE 2015 World Congress $\mathcal{E}$ Exhibition; SAE International: Brooklyn, MI, USA, 2015; doi:10.4271/2015-01-1538. [CrossRef]

6. Ashton, N.; West, A.; Lardeau, S.; Revell, A. Assessment of RANS and DES methods for realistic automotive models. Comput. Fluids 2016, 128, 1-15. [CrossRef]

7. Buscariolo, F.F.; Assi, G.R.; Sherwin, S.J. Computational study on an Ahmed Body equipped with simplified underbody diffuser. J. Wind Eng. Ind. Aerodyn. 2021, 209, 104411. [CrossRef]

8. Ahmed, S.; Ramm, G.; Faltin, G. Some Salient Features Of The Time-Averaged Ground Vehicle Wake. In SAE Technical Paper; SAE International: Brooklyn, MI, USA, 1984; doi:10.4271/840300. [CrossRef]

9. Le Good, G.M.; Garry, K.P. On the Use of Reference Models in Automotive Aerodynamics. In SAE 2004 World Congress $\mathcal{E}$ Exhibition; SAE International: Brooklyn, MI, USA, 2004; doi:10.4271/2004-01-1308. [CrossRef]

10. Heft, A.; Indinger, T.; Adams, N. Investigation of Unsteady Flow Structures in the Wake of a Realistic Generic Car Model. In Proceedings of the 29th AIAA Applied Aerodynamics Conference, Honolulu, HI, USA, 27-30 June 2011; doi:10.2514/6.2011-3669. [CrossRef]

11. Heft, A.I.; Indinger, T.; Adams, N.A. Experimental and Numerical Investigation of the DrivAer Model. Symposia, Parts A and B Fluids Eng. Div. Summer Meet. 2012, 1, 41-51. [CrossRef]

12. Aljure, D.; Calafell, J.; Baez, A.; Oliva, A. Flow over a realistic car model: Wall modeled large eddy simulations assessment and unsteady effects. J. Wind Eng. Ind. Aerodyn. 2018, 174, 225-240. [CrossRef]

13. Rüttgers, M.; Park, J.; You, D. Large-eddy simulation of turbulent flow over the DrivAer fastback vehicle model. J. Wind Eng. Ind. Aerodyn. 2019, 186, 123-138. [CrossRef]

14. Bassi, F.; Botti, L.; Colombo, A.; Crivellini, A.; Franciolini, M.; Ghidoni, A.; Noventa, G. A p-adaptive Matrix-Free Discontinuous Galerkin Method for the Implicit LES of Incompressible Transitional Flows. Flow Turbul. Combust. 2020, 105, 437-470. [CrossRef]

15. Grinstein, F.F.; Margolin, L.G.; Rider, W.J. Implicit Large Eddy Simulation; Cambridge University Press: Cambridge, UK, 2007; Volume 10

16. Uranga, A.; Persson, P.O.; Drela, M.; Peraire, J. Implicit Large Eddy Simulation of transition to turbulence at low Reynolds numbers using a Discontinuous Galerkin method. Int. J. Numer. Methods Eng. 2011, 87, 232-261. [CrossRef]

17. de Wiart, C.; Hillewaert, K.; Bricteux, L.; Winckelmans, G. Implicit LES of free and wall-bounded turbulent flows based on the discontinuous Galerkin/symmetric interior penalty method. Int. J. Numer. Methods Fluids 2015, 78, 335-354. [CrossRef]

18. Moura, R.; Sherwin, S.; Peiró, J. Linear dispersion-diffusion analysis and its application to under-resolved turbulence simulations using discontinuous Galerkin spectral/hp methods. J. Comput. Phys. 2015, 298, 695-710. [CrossRef]

19. Bassi, F.; Botti, L.; Colombo, A.; Crivellini, A.; Ghidoni, A.; Massa, F. On the development of an implicit high-order Discontinuous Galerkin method for DNS and implicit LES of turbulent flows. Eur. J. Mech. B/Fluids 2016, 55, 367-379. [CrossRef]

20. de la Llave Plata, M.; Couaillier, V.; le Pape, M.C. On the use of a high-order discontinuous Galerkin method for DNS and LES of wall-bounded turbulence. Comput. Fluids 2018, 176, 320-337. [CrossRef]

21. Moura, R.; Mengaldo, G.; Peiró, J.; Sherwin, S. On the eddy-resolving capability of high-order discontinuous Galerkin approaches to implicit LES/under-resolved DNS of Euler turbulence. J. Comput. Phys. 2017, 330, 615-623. [CrossRef]

22. Park, J.; Witherden, F.; Vincent, P. High-order implicit large-eddy simulations of flow over a NACA0021 aerofoil. AIAA J. 2017, 55, 2186-2197. [CrossRef]

23. Bassi, F.; Botti, L.; Colombo, A.; Crivellini, A.; Ghidoni, A.; Nigro, A.; Rebay, S. Time Integration in the discontinuous Galerkin Code MIGALE - Unsteady Problems. In IDIHOM: Industrialization of High-Order Methods-A Top-Down Approach; Notes on Numerical Fluid Mechanics and Multidisciplinary Design; Kroll, N., Hirsch, C., Bassi, F., Johnston, C., Hillewaert, K., Eds.; Springer International Publishing: Berlin/Heidelberg, Germany; Springer Nature Switzerland AG: Cham, Switzerland, 2015; Volume 128, pp. 205-230. 
24. Garai, A.; Diosady, L.; Murman, S.; Madavan, N. Scale-resolving simulations of bypass transition in a high-pressure turbine cascade using a spectral element discontinuous Galerkin Method. J. Turbomach. 2018, 140. [CrossRef]

25. Bassi, F.; Colombo, A.; Crivellini, A.; Fidkowski, K.; Franciolini, M.; Ghidoni, A.; Noventa, G. An entropy-adjoint $p$-adaptive discontinuous Galerkin method for the under-resolved simulation of turbulent flows. In Proceedings of the AIAA Aviation 2019 Forum, Dallas, TX, USA, 17-21 June 2019; doi:10.2514/6.2019-3418. [CrossRef]

26. Franciolini, M.; Botti, L.; Colombo, A.; Crivellini, A. p-Multigrid matrix-free discontinuous Galerkin solution strategies for the under-resolved simulation of incompressible turbulent flows. Comput. Fluids 2020, 206, 104558. [CrossRef]

27. Noventa, G.; Massa, F.; Bassi, F.; Colombo, A.; Franchina, N.; Ghidoni, A. A high-order Discontinuous Galerkin solver for unsteady incompressible turbulent flows. Comput. Fluids 2016, 139, 248-260. [CrossRef]

28. Lang, J.; Verwer, J. ROS3P-An accurate third-order Rosenbrock solver designed for parabolic problems. BIT Numer. Math. 2001, 41, 731-738.:1021900219772. [CrossRef]

29. Bassi, F.; Botti, L.; Colombo, A.; Ghidoni, A.; Massa, F. Linearly implicit Rosenbrock-type Runge-Kutta schemes applied to the Discontinuous Galerkin solution of compressible and incompressible unsteady flows. Comput. Fluids 2015, 118, 305-320. [CrossRef]

30. Massa, F.; Noventa, G.; Lorini, M.; Bassi, F.; Ghidoni, A. High-order linearly implicit two-step peer schemes for the discontinuous Galerkin solution of the incompressible Navier-Stokes equations. Comput. Fluids 2018, 162, 55-71. [CrossRef]

31. Noventa, G.; Massa, F.; Rebay, S.; Bassi, F.; Ghidoni, A. Robustness and efficiency of an implicit time-adaptive discontinuous Galerkin solver for unsteady flows. Comput. Fluids 2020, 204, 104529. [CrossRef]

32. Naddei, F.; de la Llave Plata, M.; Couaillier, V.; Coquel, F. A comparison of refinement indicators for $p$-adaptive simulations of steady and unsteady flows using discontinuous Galerkin methods. J. Comput. Phys. 2019, 376, 508-533. [CrossRef]

33. Bassi, F.; Colombo, A.; Crivellini, A.; Franciolini, M.; Ghidoni, A.; Manzinali, G.; Noventa, G. Under-resolved simulation of turbulent flows using a $p$-adaptive discontinuous Galerkin method. Springer Proc. Phys. 2019, 226, 157-162._25. [CrossRef]

34. Tugnoli, M.; Abbà, A.; Bonaventura, L.; Restelli, M. A locally $p$-adaptive approach for Large Eddy Simulation of compressible flows in a DG framework. J. Comput. Phys. 2017, 349, 33-58. [CrossRef]

35. Bassi, F.; Colombo, A.; Crivellini, A.; Fidkowski, K.J.; Franciolini, M.; Ghidoni, A.; Noventa, G. Entropy-Adjoint $p$-Adaptive Discontinuous Galerkin Method for the Under-Resolved Simulation of Turbulent Flows. AIAA J. 2020, 58, 3963-3977. [CrossRef]

36. Hartmann, R.; Balan, A.; Bassi, F.; Boussuge, J.F.; de Brauer, A.; Cagnone, J.S.; Colombo, A.; Couaillier, V.; Coulaud, O.; Crivellini, A.; et al. Space Adaptive Methods/Meshing. In TILDA: Towards Industrial LES/DNS in Aeronautics: Paving the Way for Future Accurate CFD—Results of the H2020 Research Project TILDA, Funded by the European Union, 2015-2018; Hirsch, C., Hillewaert, K., Hartmann, R., Couaillier, V., Boussuge, J.F., Chalot, F., Bosniakov, S., Haase, W., Eds.; Springer International Publishing: Cham, Switzerland, 2021; pp. 103-190._4. [CrossRef]

37. Krivodonova, L.; Xin, J.; Remacle, J.F.; Chevaugeon, N.; Flaherty, J.E. Shock detection and limiting with discontinuous Galerkin methods for hyperbolic conservation laws. Appl. Numer. Math. 2004, 48, 323-338. [CrossRef]

38. Gassner, G.; Staudenmaier, M.; Hindenlang, F.; Atak, M.; Munz, C.D. A space-time adaptive discontinuous Galerkin scheme. Comput. Fluids 2015, 117, 247 - 261. [CrossRef]

39. Persson, P.O.; Peraire, J. Sub-cell shock capturing for discontinuous Galerkin methods. In Proceedings of the 44th AIAA Aerospace Sciences Meeting and Exhibit, Reno, NV, USA, 9-12 January 2006; p. 112.

40. GridPro Website. Available online: https:/ /www.gridpro.com/ (accessed on 9 December 2019).

41. Frank, T.; Gerlicher, B.; Abanto, J. DrivAer-Aerodynamic Investigations for a New Realistic Generic Car Model using ANSYS CFD. In Proceedings of the ASWC 2013-Automotove Simulation World Congress, Frankfurt am Main, Germany, 29-30 October 2013. 